Penultimate draft of the paper appearing in

A. Baltag, J. Seligman, and T. Yamada (eds.) Logic, Rationality, and Interaction: 6th International Workshop, Berlin: Springer, pp. 268-283

The final publication is available at Springer via

http://dx.doi.org/10.1007/978-3-662-55665-8_19

\title{
Conjunction and Disjunction in Infectious Logics
}

\author{
Hitoshi Omori ${ }^{1}$ and Damian Szmuc ${ }^{2,3}$ \\ ${ }^{1}$ Department of Philosophy, Kyoto University, Japan. \\ ${ }^{2}$ IIF-SADAF, National Scientific and Technical Research Council (CONICET), Argentina \\ ${ }^{3}$ Department of Philosophy, University of Buenos Aires, Argentina
}

\begin{abstract}
In this paper we discuss the extent to which conjunction and disjunction can be rightfully regarded as such, in the context of infectious logics. Infectious logics are peculiar manyvalued logics whose underlying algebra has an absorbing or infectious element, which is assigned to a compound formula whenever it is assigned to one of its components. To discuss these matters, we review the philosophical motivations for infectious logics due to Bochvar, Halldén, Fitting, Ferguson and Beall, noticing that none of them discusses our main question. This is why we finally turn to the analysis of the truth-conditions for conjunction and disjunction in infectious logics, employing the framework of plurivalent logics, as discussed by Priest. In doing so, we arrive at the interesting conclusion that -in the context of infectious logics - conjunction is conjunction, whereas disjunction is not disjunction.
\end{abstract}

\section{Introduction}

\subsection{Background, motivation and aim}

The aim of this paper is to discuss the extent to which conjunctions and disjunctions, appearing in the context of what are nowadays called infectious logics (cf. [13, 22, 31]), can be rightfully called conjunction and disjunction. Infectious logics are, in a nutshell, non-classical many-valued logics that count with a truth-value which is assigned to a compound formula every time it is assigned to at least one of its components. Thus, it is claimed that values behaving in this way exhibit an infectious, contaminating or otherwise absorbing nature.

Salient examples of such logics are the $\{\neg, \wedge, \vee\}$-fragments, also called the "classical" fragments, of Dmitri Bochvar's and Sören Halldén's logic of nonsense, presented in e.g. [5] and [18] 
respectively. What differentiates these logics (or, properly speaking, their classical fragments) is that while Bochvar treats the contaminating value as undesignated, Halldén (although derivatively, cf. [12, p. 345] and [22]) treats it as designated. From this and the absorbing nature of this element it can be inferred that Bochvar's logic is paracomplete, whereas Halldén's logic is paraconsistent. In fact, the classical fragment of Bochvar's logic has been also discussed in the relevant literature as Weak Kleene Logic $\mathbf{K}_{\mathbf{3}}^{\mathbf{w}}$, while the classical fragment of Halldén's logic has been also independently discussed in the literature as Paraconsistent Weak Kleene Logic PWK. As is easy to notice and as has been already pointed out in many works (e.g. 29]) this logics are such that, respectively, $\vee$-Introduction and $\wedge$-Elimination are invalid in them.

To carry out our present discussion we will scrutinize various motivations given for these infectious logics, in order to determine whether or not the target binary operations are, after all, legitimate disjunctions and conjunctions. To this end, for the case of paracomplete infectious logics, we will consider Bochvar's own nonsense-related account, Melvin Fitting's epistemic semantics [16], Thomas Ferguson's computational interpretation [12, 14, and Jc Beall's off-topic reading 2 . Whereas for the case of paraconsistent infectious logics, we will consider Halldén's own nonsense-related account. We will argue that none of these allow to present a cogent reading of disjunction and conjunction, but that an alternative account of the truth and falsity conditions for these connectives, in terms of the discussion of Graham Priest's plurivalent semantics carried out in [29, 22], indeed does the work.

\subsection{Preliminaries}

Our language $\mathcal{L}$ consists of a finite set $\{\neg, \wedge, \vee\}$ of propositional connectives and a countable set Prop of propositional parameters. Furthermore, we denote by Form the set of formulas defined as usual in $\mathcal{L}$. We denote formulas of $\mathcal{L}$ by $\alpha, \beta, \gamma$, etc. and sets of formulas of $\mathcal{L}$ by $\Gamma, \Delta, \Sigma$, etc.

Definition 1.1 (Univalent semantics) $A$ univalent semantics for the language $\mathcal{L}$ is a structure $M=\langle\mathcal{V}, \mathcal{D}, \delta\rangle$, where

- $\mathcal{V}$ is a non-empty set of truth values,

- $\mathcal{D}$ is a non-empty proper subset of $\mathcal{V}$, the designated values,

- for every $n$-ary connective $*$ in the language, $\delta_{*}: \mathcal{V}^{n} \rightarrow \mathcal{V}$ is the truth function for $*$

A univalent interpretation is a pair $\langle M, \mu\rangle$, where $M$ is such a structure, and $\mu$ is an evaluation function from the Prop to $\mathcal{V}$. Given an interpretation, $\mu$ is extended to a map from all formulas to $\mathcal{V}$ recursively: $\mu\left(*\left(\alpha_{1}, \ldots, \alpha_{n}\right)\right)=\delta_{*}\left(\mu\left(\alpha_{1}\right), \ldots, \mu\left(\alpha_{n}\right)\right)$. Finally, $\Sigma \models_{u}^{M} \alpha$ iff in every interpretation in which all the formulas of $\Sigma$ are designated, so is $\alpha \square$

Note that semantic consequence relations are defined as preservation of designated values, as usual. For an alternative, see Definitions 1.3 and 1.4

Definition 1.2 The univalent semantics for Weak Kleene and Paraconsistent Weak Kleene for the language $\mathcal{L}$ are the structures $M_{\mathbf{K}_{3}^{\mathrm{w}}}=\left\langle\mathcal{V}_{\mathbf{K}_{3}^{\mathrm{w}}}, \mathcal{D}_{\mathbf{K}_{3}^{\mathrm{w}}}, \delta_{\mathbf{K}_{3}^{\mathrm{w}}}\right\rangle$, and $M_{\mathbf{P W K}}=\left\langle\mathcal{V}_{\mathbf{P W K}}, \mathcal{D}_{\mathbf{P W K}}, \delta_{\mathbf{P W K}}\right\rangle$ respectively where

$$
\text { - } \mathcal{V}_{\mathbf{K}_{3}^{\mathrm{w}}}=\mathcal{V}_{\mathbf{P W K}}=\{\mathbf{t}, \mathbf{e}, \mathbf{f}\}
$$

\footnotetext{
${ }^{1}$ We will sometimes omit the subscript $u$, when contexts disambiguates. Also, we may sometimes make reference of $\models^{\mathbf{L}}$ instead of $\models^{M_{\mathbf{L}}}$
} 
- $\mathcal{D}_{\mathbf{K}_{\mathbf{3}}^{\mathrm{w}}}=\{\mathbf{t}\}$ and $\mathcal{D}_{\mathbf{P W K}}=\{\mathbf{t}, \mathbf{e}\}$,

- $\delta_{\mathbf{K}_{3}^{\mathrm{w}}}=\delta_{\mathbf{P W K}}$ and is the set of truth-functions represented by Kleene's 'weak' truth-tables from [19], depicted below.

\begin{tabular}{|c|c|c|c|c|c|c|c|c|c|}
\hline & $\delta_{\neg}$ & $\delta_{\wedge}$ & $\mathbf{t}$ & e & f & $\delta_{\vee}$ & $\mathbf{t}$ & $\mathbf{e}$ & $\mathbf{f}$ \\
\hline $\mathbf{t}$ & $\mathbf{f}$ & $t$ & $\mathbf{t}$ & $\mathbf{e}$ & $f$ & $\mathbf{t}$ & $\mathbf{t}$ & $\mathbf{e}$ & $\mathbf{t}$ \\
\hline $\mathbf{e}$ & $\mathbf{e}$ & $\mathbf{e}$ & $\mathbf{e}$ & $\mathbf{e}$ & $\mathbf{e}$ & $\mathbf{e}$ & $\mathbf{e}$ & $\mathbf{e}$ & $\mathbf{e}$ \\
\hline $\mathbf{f}$ & $\mathbf{t}$ & $\mathbf{f}$ & f & $\mathbf{e}$ & f & f & $\mathbf{t}$ & $\mathbf{e}$ & $\mathbf{f}$ \\
\hline
\end{tabular}

As is pointed out in 22], $\mathbf{K}_{\mathbf{3}}^{\mathbf{w}}$ can be understood as a logic with gaps endowed with a characterization of logical consequence in terms of truth-preservation, whereas PWK can be understood as a logic with gaps endowed with a characterization of logical consequence in terms of non-falsity preservation. The corresponding induced consequence relations being $\models^{\mathbf{K}_{3}^{\mathrm{w}}}$ and $\models^{\mathbf{P W K}}$.

Nevertheless, it is interesting to notice that these possibilities do not exhaust the way in which we can define logical consequence and, thus, in which we can build logical systems out of the weak truth-tables from Kleene. We define below a $q$-consequence relation and a $p$-consequence relation, following the proposals of Grzegorz Malinowksi in [20, and Szymon Frankowski in [17. respectively.

Definition 1.3 (q-consequence for Kleene's weak truth-tables) $\Sigma \models_{q}^{\mathbf{W K}} \alpha$ iff in every interpretation in which all the formulae of $\Sigma$ are assigned a value in $\{\mathbf{t}, \mathbf{e}\}$, then $\alpha$ is assigned the value $\mathbf{t}$.

Definition 1.4 ( $p$-consequence for Kleene's weak truth-tables) $\Sigma \models_{p}^{\mathbf{W K}} \alpha$ iff in every interpretation in which all the formulae of $\Sigma$ are assigned the value $\mathbf{t}$, then $\alpha$ is assigned a value in $\{\mathbf{t}, \mathbf{e}\}$.

\section{Infectious logics: an overview}

As we will briefly see, Weak Kleene and Paraconsistent Weak Kleene are members of a broader family of infectious logics. Intuitively, infectious logics are many-valued logics that have an absorbent or infectious truth-value, that is, a truth-value such that it is assigned to a compound formula whenever it is assigned to at least one of its components. More formally:

Definition 2.1 A semantics $M=\langle\mathcal{V}, \mathcal{D}, \delta\rangle$ for the language $\mathcal{L}$ is infectious iff there is an element $x \in \mathcal{V}$ such that for every $n$-ary connective $*$ in the language, with an associated truthfunction $\delta_{*} \in \delta$ and for all $v_{1}, \ldots, v_{n} \in \mathcal{V}$ it holds that: if $x \in\left\{v_{1}, \ldots, v_{n}\right\}$, then $\delta_{*}\left(v_{1}, \ldots, v_{n}\right)=$ $x$

It is easy to see, as has been noticed in e.g. 29, that when the infectious value in question does not belong to the set of designated values, then the logic is paracomplete. By this we mean that there is a valuation such that both $A$ and $\neg A$ are undesignated. Moreover, in these cases, yet another characteristic classical inference is invalid, namely $\vee$-Introduction (sometimes also called 'Addition'), i.e. $\varphi \vDash \varphi \vee \psi$. By this we mean that there is a valuation such that $\varphi$ is designated, but $\varphi \vee \psi$ is undesignated. This happens, particularly, when $\psi$ receives the infectious undesignated value in question $2^{2}$

\footnotetext{
${ }^{2}$ Notice that this does not suggest that the infectious value does not belong to the set of designated values if and only if the logic is paracomplete, for there might well exist paracomplete logics which do not count with an infectious value at all, as in e.g. the well-known Strong Kleene logic $\mathbf{K}_{\mathbf{3}}$ (cf. [19]).
} 
Additionally, it is also easy to see, as has been noticed in e.g. [29], that when the infectious value in question belong to the set of designated values, then the logic is paraconsistent. By this we mean that there is a valuation such that both $A$ and $\neg A$ are designated. Moreover, in these cases, yet another characteristic classical inference is invalid, namely $\wedge$-Elimination (sometimes also called 'Simplification'), i.e. $\varphi \wedge \psi \vDash \varphi$. By this we mean that there is a valuation such that $\varphi \wedge \psi$ is designated, but $\varphi$ is undesignated. This happens, particularly, when $\psi$ receives the infectious designated value in question 3

However, these logical behavior could be found to be rather odd and, for this reason, we provide an overview of the philosophical motivations they have received in the literature, in the next subsections. Let us notice that being faithful with the literature will require us reflecting the fact that a considerable amount of motivations have been discussed with regard to the paracomplete case, whereas only a few have been proposed for the paraconsistent case ${ }^{4}$

\subsection{Paracomplete case}

Bochvar's Logic of Nonsense. In the early decades of the last century, paradoxes of set theory devoured the attention of many philosophers and logicians. The first conceptual motivation for an infectious logic relates to these topics. Dmitri Bochvar developed in [5] a three-valued logic to handle the paradoxes of set theory, like Russell's Paradox (cf. [27]).

Bochvar's own take on this issue was that the sentence describing such a paradoxical sets was, properly speaking, meaningless or nonsensical and - as such - it did not deserve to be regarded as either true or false. In more contemporary terms, we would say that Bochvar took such sentences to be truth value gaps (cf. 27]).

More importantly, Bochvar was of the idea that sentences or statements containing a meaningless part or subsentence must be, in turn, meaningless themselves. Thus, meaninglessness can be legitimately described as the pathology from which paradoxical sentences suffered, which is indeed itself literally infectious. Furthermore, since the meaninglessness of these very sentences is portrayed by Bochvar via the assignment of the corresponding non-classical value we must say that, in terms of Definition 2.1, the meaningless value is infectious.

These motivations led Bochvar to devise his 'logic of nonsense', which besides the "classical" connectives, has also means to mark nonsensical or meaningless statements. The 'external assertion' operator acts like a characteristic function for true statements, i.e. statements assigned the value and, therefore, not assigned the value false, or the meaningless value. To be precise, then, it is only the $\{\neg, \wedge, \vee\}$-fragment of Bochvar's logic of nonsense that represents an infectious logic (also found in the literature as Weak Kleene Logic $\mathbf{K}_{\mathbf{3}}^{\mathbf{w}}$, on which more below).

Finally, Bochvar took logical consequence as being characterized by truth-preservation. That is, necessarily, if the premises are true, the conclusion is true. But, again, if meaningless sentences are neither true nor false, then an inference with true premises but a meaningless conclusion must be invalid. This is why, by taking e.g. $\varphi$ to be true and $\psi$ to be meaningless, $\vee$-Introduction fails.

Fitting's Epistemic Interpretation. With the intention of applying his project (see e.g. [16]) of providing an epistemic interpretation for Kleene logics (cf. [19]) and Belnap-Dunn four-

\footnotetext{
${ }^{3}$ Analogous to the previous footnote, notice that this does not suggest that the infectious value does belong to the set of designated values if and only if the logic is paraconsistent, for there might well exist paraconsistent logics which do not count with an infectious value at all, as in e.g. the Logic of Paradox due to Priest (cf. [27]).

${ }^{4}$ We should remark that providing a full overview of these motivations will require much more space than we have here. For that reason, we refrained from commenting on some of the motivations for infectious logics, e.g. (the first degree of) Parry systems (cf. [25]) and of Epstein's Dependence and Dual Dependence systems (cf. [11) discussed in e.g. 23] 24, 12, 14, 13, Deutsch's logic from [9], Daniels' logic from [7, and Priest's logic $\mathbf{F D E}_{\varphi}$ from 28.
} 
valued logic FDE, Melvin Fitting provided in [15] an epistemic interpretation for $\mathbf{K}_{\mathbf{3}}^{\mathbf{w}}$. For this purpose, he recurred to the framework present in e.g. [16, where there is a set of experts expressing their positive and negative opinion on different issues, represented by sentences $\varphi, \psi$, etc. In his discussion, and taking $\varphi$ as an example, Fitting thought of allowing experts to be for (and not against) $\varphi$, or both for and against $\varphi$, or neither for nor against $\varphi$, or against (and not for) $\varphi$. These four cases correspond, respectively, (via a suitable translation) to the four values $\{\mathbf{t}, \mathbf{b}, \mathbf{n}, \mathbf{f}\}$ of $\mathbf{F D E}$.

In this framework, it is possible to ask (when all experts have made their minds about the relevant issues) which is e.g. the set of experts that are in favor of $\varphi \vee \psi$. In this case, we might think of the union of those in favor of $\varphi$ and those in favor of $\psi$. However, Fitting notices that in some situations of this sort we might want to cut-down the set of experts taken into account to those who have actually expressed an opinion towards both $\varphi$ and $\psi$. That is, there can be some situations (Fitting argues) where we may not want to count an expert as being in favor of $\varphi \vee \psi$, if she has no opinion at all with regard to e.g. $\psi$. In those cases we are interested, in Fitting's terminology, in a 'cut-down' disjunction. (And, by similar remarks, in a 'cut-down' negation and a 'cut-down' conjunction).

The failure of $\vee$-Introduction is, thus, properly understood in epistemic terms by taking disjunction as cut-down disjunction. It is not the case that from e.g. the fact that all experts are in favor of $\varphi$ it follows that all experts are in favor of $\varphi \vee \psi$, for some experts may have no opinion whatsoever with regard to $\psi$. If we, additionally, are in a situation where no expert is both in favor and against a certain issue (that is, if no sentence is assigned the truth-value $\mathbf{b}$ ), the logic induced by these cut-down operations is, precisely, $\mathbf{K}_{\mathbf{3}}^{\mathbf{w}}$.

Ferguson's Computational Interpretation. In [12] Thomas Ferguson advances a computational reading of some paracomplete infectious logics by following Belnap's classical remarks about how a computer should think (cf. [4), using FDE. Belnap motivates his system by considering a computer retrieving information about certain sentences, where this information can be thought as the truth-value that - the computer is told - the given sentences have. Belnap imagines that, with regard to e.g. $\varphi$, the computer can be told, i.e. it can retrieve the information that $\varphi$ is true, or that $\varphi$ is false, or both, or neither ${ }^{5}$

Ferguson's 'faulty computer' approach to infectious logics focuses on the idea that a computer may fail to retrieve the value of a given sentence. He notices, moreover, that this case must me taken to be essentially different from that where the computer is able to retrieve the value of $\varphi$, but it encounters no information regarding its truth or its falsity. The case of a failure retrieving the target value, possibly caused by a memory crash, a physical malfunction, or other problem, is thus different from the case of a successful retrieving attempt, accompanied by the fact that the target value contains no information.

Failures, in Ferguson's approach, must be represented (in an extension of FDE) by a fifth value, behaving infectiously and being undesignated. In such a case, $\vee$-Introduction is invalid, for -although a computer might be successful in retrieving the value of $\varphi$ and, additionally, being told that it is true - it might encounter a critical error or a crash while retrieving the value of $\psi$ and, therefore, an error while retrieving the value of $\varphi \vee \psi 6$

Beall's Off-Topic Interpretation. In 2, Jc Beall proposes an alternative interpretation for Weak Kleene Logic, which does not appeal to meaninglessness, as Bochvar's does. Beall focuses on theories formulated in English (or any other natural language). Theories have a distinctive topic, that is, they are not about everything, namely, about every concept expressible in English.

\footnotetext{
${ }^{5} \mathrm{As}$ is noted in [12, while this framework is regarded as a 'single address' approach to Belnap computers, a 'two address' approach can also motivated, with the subtlety that it induces a weaker nine-valued logic.

${ }^{6}$ We shall also mention that in [14] Ferguson discusses another computational interpretation related to McCarthy's logic from 21].
} 
Intuitively, color theory is about colors, arithmetic is about numbers, and so on and so forth, but color theory is not about numbers and arithmetic is not about colors. To these assumptions Beall adds the intuitive thesis that $\varphi \vee \psi$ is about what $\varphi$ is about and about what $\psi$ is about, and similarly for $\neg \varphi$ and $\varphi \wedge \psi$.

But this is not all that can be said about theories. Theories, the standard view goes, are sets of sentences closed under logical consequence. But the main question is: which logic? Beall remarks that it cannot be a logic that validates $\vee$-Introduction. For, if that is the case, then any theory that is about what $\varphi$ is about, will end up being also about what $\psi$ is about, even if $\psi$ is completely off-topic. But this is unintuitive, for then theories will be about every everything, that is, about every concept expressible in English.

To this extent, Beall proposes that the logic under which theories should be closed should be Weak Kleene. By doing this, he also proposes to interpret the infectious value as off-topic, thereby understanding validity as on-topic truth preservation. It is easy to see how this invalidates $\vee$-Introduction, for even if $\varphi$ and $\psi$ are both true, $\varphi$ might well be on-topic while $\psi$ is not. Therefore, $\varphi \vee \psi$ will be true, although off-topic, whence the failure of the corresponding inference.

\subsection{Paraconsistent case}

Halldén's Logic of Nonsense. In a similar path than Bochvar, we can find Halldén's own 'logic of nonsense', developed mainly in [18. Besides the usual set-theoretic paradoxes, Halldén also finds instances of meaningless or nonsensical sentences involved in paradoxes of vagueness (cf. [27]). Again, just like Bochvar, Halldén takes meaningless or nonsensical statements to be neither true nor false, and therefore to be truth-value gaps. Halldén also shares the idea that meaninglessness is an infectious feature.

These motivations led him, in turn, to conceive his own 'logic of nonsense', which besides the usual logical connectives $\neg, \wedge, \vee$, has a 'meaningfulness operator' that acts like a characteristic function for meaningful statements, i.e. statements assigned either truth or falsity. To be precise, then, it is only the $\{\neg, \wedge, \vee\}$-fragment of Halldén's logic of nonsense that represents an infectious logic (also found in the literature as PWK, on which more below).

Bochvar and Halldén's logics of nonsense have, nevertheless, an important difference. Whereas the first is paracomplete, the latter is paraconsistent. Formally speaking, this means that the truth-value assigned to meaningless sentences is regarded as designated. Thus, there are sentences $A$ (namely, meaningless sentences and sentences containing meaningless statements as subsentences) such that both $A$ and $\neg A$ are designated.

With regard to this, while it is argued in [6] that these renders the meaningless value as being truth-like, a more comprehensive understanding of this feature can be taken into account if we notice that paraconsistency, in Halldén's case, is a derivative phenomenon. This means that Halldén did not think of nonsensical sentences as being both true and false, i.e. as truth-value gluts (cf. [27]), much to the contrary he took them to neither true nor false.

The paraconsistent nature of the induced consequence relation is, therefore, better understood if we point out (as [12, p. 345] and [22] do) that Halldén should be regarded as taking validity to be characterized by (forwards) non-falsity preservation, that is, if the premises are non-false, then the conclusion is non-false - instead of the usual (forwards) truth-preservation.77 In this vein, if meaningless sentences are neither true nor false, then an inference with meaningless premises but a false conclusion must be invalid. This is why, by taking e.g. $\varphi$ to be false and $\psi$ to be meaningless, $\wedge$-Elimination fails.

\footnotetext{
${ }^{7}$ Non-falsity preservation as a motivation for paraconsistency in 'gappy' contexts is discussed in e.g. [3]
} 
Dualizing Fitting's Epistemic Interpretation $\sqrt{8}$ It is possible to conceive two dualizations of Fitting's epistemic understanding of Weak Kleene logic $\mathbf{K}_{\mathbf{3}}^{\mathbf{w}}$, which will provide an epistemic interpretation of a our target paraconsistent infectious logic, namely, PWK. The first one takes the entire framework of Fitting's cut-down operations, but changes the way the consequence relation is defined. Instead of taking validity to be defined by truth-preservation, we change to define it as non-falsity preservation. By this we mean that an inference is valid if and only if if the premises are not taken to be false by all the experts, then the conclusion is not taken to be false by all the experts. In such cases, the failure of $\wedge$-Elimination is properly understood in epistemic terms by taking conjunction as cut-down conjunction. Imagine a situation where all experts have no opinion towards $\varphi$ : in such a situation it is not the case that from e.g. the fact that all experts have no opinion towards $\varphi \wedge \psi$ it follows that all experts have no opinion towards $\psi$, for all experts may have a negative opinion towards $\psi$. If we, additionally, are in a situation where no expert is both in favor and against a certain issue (that is, if no sentence is assigned the truth-value $\mathbf{b}$ ), the logic induced by these cut-down operations, taking validity to be defined by non-falsity preservation, is precisely $\mathbf{P W K}$.

Alternatively, we could take Fitting's epistemic understanding of FDE and build a different interpretation for PWK. Again, in this framework, it is possible to ask (when all experts have made their minds about the relevant issues) which is e.g. the set of experts that are in favor of $\varphi \wedge \psi$. In this case, we might think of the intersection of those in favor of $\varphi$ and those in favor of $\psi$. However, we might be interested in some situations of this sort we might want to track-down those experts who have actually expressed an inconsistent opinion towards either $\varphi$ or $\psi$. That is, there can be some situations where we may not want to count an expert as having a consistent opinion towards e.g. $\varphi \wedge \psi$, if she has an inconsistent opinion towards e.g. $\psi$. In those cases we are interested, in analogy with Fitting's terminology, in a 'track-down' disjunction. (And, by similar remarks, in a 'track-down' negation and a 'track-down' conjunction).

Now, imagine a situation where all experts have a negative opinion towards $\varphi$ and all experts have an inconsistent opinion towards $\psi$-i.e. they are both for and against $\psi$. Thus, following the track-down policy we would say that all experts have, therefore, an inconsistent opinion towards $\varphi \wedge \psi$. The failure of $\wedge$-Elimination is, thus, properly understood in epistemic terms by taking conjunction as track-down conjunction. In such a situation, it is not the case that from e.g. the fact that all experts are both in favor and against of $\varphi \wedge \psi$ it follows that all experts are in favor of $\varphi$, for experts may be both for and against the conjunction just because they have an inconsistent opinion towards $\psi$. If we, additionally, are in a situation where no expert is silent regarding all issues (that is, if no sentence is assigned the truth-value $\mathbf{n}$ ), the logic induced by these track-down operations is, precisely, PWK.

\section{Plurivalent semantics: basics}

We would like to remark that, even if all of the above formalisms involve infectious connectives that are referred in their respective contexts as conjunction and disjunction, it is never discussed in these works if the target operations are actual conjunctions and disjunctions, or what makes them be so.

Our discussion in the sections to come is intended to answer this question, by looking at the truth and falsity conditions of conjunction and disjunction. We will do this within the framework of plurivalent logics developed by Priest in 29. Plurivalent logics and their semantics can be

\footnotetext{
${ }^{8}$ Unlike the previous interpretations of both the paracomplete and the paraconsistent infectious systems, the following account is our original thought. We would like to thank one of the reviewers for the suggestion to develop further the epistemic readings of infectious logics. For a full technical development of these ideas, see 30.
} 
thought as an alternative way to look at logical frameworks where instead of a formula's single truth-value coming from an arbitrary set, it is allowed for formulae to have more than one truth-value, from a given set. Thus, for example, a setting in which each formulae gets, as a truth-value, a single element of $\{\mathbf{t}, \mathbf{e}, \mathbf{f}\}$, can also be represented in a setting in which every formulae gets, as a truth-value, a subset of $\{\mathbf{t}, \mathbf{f}\}$.

The definitions and results in the first three subsections are all given by Priest in [29] in which proofs are fully spelled out. Moreover, the definitions and results in the last subsection can be found in [22]. Therefore, the results in this section are stated without proofs. Note finally, that our notation as well as the order of the presentation are slightly different from Priest's $9^{9}$

\subsection{General plurivalent semantics.}

We begin with the most general case of plurivalent semantics.

Definition 3.1 (General plurivalent semantics) Given a univalent interpretation, the corresponding general plurivalent interpretation is the same, except that it replaces the evaluation function, $\mu$, with a one-many evaluation relation, $\mathfrak{R}$, between Prop and $\mathcal{V}$. Given an interpretation, $\mathfrak{R}$ is extended to a map from Form to $\mathcal{V}$ recursively:

$$
*\left(\alpha_{1}, \ldots, \alpha_{n}\right) \mathfrak{R} v \text { iff for some } v_{1}, \ldots, v_{n}:\left(\alpha_{i} \mathfrak{R} v_{i} \text { and } v=\delta_{*}\left(v_{1}, \ldots, v_{n}\right)\right)
$$

Finally, $\Sigma=_{g}^{M} \alpha$ iff for all $\mathfrak{R}$, if $\mathfrak{R}$ designates all the formulas of $\Sigma$ then $\mathfrak{R}$ designates $\alpha$, where $\mathfrak{R}$ designates $\alpha$ iff $\alpha \mathfrak{R} v$ for some $v \in \mathcal{D}$.

Then, we can again prove a general relation between the univalent semantics and general plurivalent semantics. To this end, we need the following definition.

Definition 3.2 Let $M=\langle\mathcal{V}, \mathcal{D}, \delta\rangle$ be a univalent semantics. Then we can define a univalent semantics $\ddot{M}=\langle\ddot{\mathcal{V}}, \ddot{\mathcal{D}}, \ddot{\delta}\rangle$, where $\ddot{\mathcal{V}}=2^{\mathcal{V}}, \ddot{\mathcal{D}}=\{\ddot{v} \in \ddot{\mathcal{V}}: v \in \ddot{v}$ for some $v \in \mathcal{D}\}$ and

$$
v \in \ddot{\delta}_{*}\left(\ddot{v}_{1}, \ldots, \ddot{v_{n}}\right) \text { iff for some } v_{1}, \ldots, v_{n}:\left(v_{i} \in \ddot{v}_{i} \text { and } v=\delta_{*}\left(v_{1}, \ldots, v_{n}\right)\right) \text {. }
$$

Proposition 3.3 Given any univalent semantics $M=\langle\mathcal{V}, \mathcal{D}, \delta\rangle$, its corresponding general plurivalent semantics can be seen as a univalent semantics $\ddot{M}=\langle\ddot{\mathcal{V}}, \ddot{\mathcal{D}}, \ddot{\delta}\rangle$, i.e. for any $\Sigma \cup\{\alpha\}$ : $\Sigma \models{ }_{g}^{M} \alpha$ iff $\Sigma \models{ }_{u}^{\ddot{M}} \alpha$

\subsection{Positive plurivalent semantics.}

We now turn to the positive plurivalent semantics, which is obtained by adding a constraint to the general plruvialent semantics. Note that the original idea behind the general construction can be found already in 26 .

Definition 3.4 (Positive plurivalent semantics) Given a univalent interpretation, the corresponding positive plurivalent interpretation is the same, except that it replaces the evaluation function, $\mu$, with a one-many evaluation relation, $\mathfrak{R}$, between Prop and $\mathcal{V}$ with the following positivity condition:

$$
\text { for every } p \in \text { Prop: } p \Re v \text { form some } v \in \mathcal{V}
$$

Given an interpretation, $\mathfrak{R}$ is extended to a map from Form to $\mathcal{V}$ recursively:

\footnotetext{
${ }^{9}$ We would like to thank one of the reviewers for the suggestion to restructure the presentation of plurivalent semantics.
} 


$$
*\left(\alpha_{1}, \ldots, \alpha_{n}\right) \Re v \text { iff for some } v_{1}, \ldots, v_{n}:\left(\alpha_{i} \Re v_{i} \text { and } v=\delta_{*}\left(v_{1}, \ldots, v_{n}\right)\right)
$$

Finally, $\Sigma=_{p}^{M} \alpha$ iff for all $\mathfrak{R}$, if $\mathfrak{R}$ designates all the formulas of $\Sigma$ then $\mathfrak{R}$ designates $\alpha$, where $\mathfrak{R}$ designates $\alpha$ iff $\alpha \mathfrak{R} v$ for some $v \in \mathcal{D}$.

Then, we can prove a general relation between the two semantics. To state the result, the following definition will be useful.

Definition 3.5 Let $M=\langle\mathcal{V}, \mathcal{D}, \delta\rangle$ be a univalent semantics. Then we can define a univalent semantics $\dot{M}=\langle\dot{\mathcal{V}}, \dot{\mathcal{D}}, \dot{\delta}\rangle$, where $\dot{\mathcal{V}}=2^{\mathcal{V}} \backslash \emptyset, \dot{\mathcal{D}}=\{\dot{v} \in \dot{\mathcal{V}}: v \in \dot{v}$ for some $v \in \mathcal{D}\}$ and

$$
v \in \dot{\delta_{*}}\left(\dot{v}_{1}, \ldots, \dot{v_{n}}\right) \text { iff for some } v_{1}, \ldots, v_{n}:\left(v_{i} \in \dot{v}_{i} \text { and } v=\delta_{*}\left(v_{1}, \ldots, v_{n}\right)\right) \text {. }
$$

Proposition 3.6 Given any univalent semantics $M=\langle\mathcal{V}, \mathcal{D}, \delta\rangle$, its corresponding positive plurivalent semantics can be seen as a univalent semantics $\dot{M}=\langle\dot{\mathcal{V}}, \dot{\mathcal{D}}, \dot{\delta}\rangle$, i.e. for any $\Sigma \cup\{\alpha\}$ : $\Sigma \models_{p}^{M} \alpha$ iff $\Sigma=_{u}^{\dot{M}} \alpha$

Remark 3.7 Let us notice, in passing, that until now our discussion of plurivalent logics has been mainly focused on logical consequence as preservation of "receiving at least one univalently designated value". But that is not the only way, as we can also think of preservation of "not receiving any univalently undesignated value", and more 10

Once we obtain the plurivalent semantics, we can also characterize the general plurivalent semantic consequence relation in terms of positive plurivalence.

Definition 3.8 Let $M=\langle\mathcal{V}, \mathcal{D}, \delta\rangle$ be a univalent semantics. Then we can define a univalent semantics $M^{e}=\left\langle\mathcal{V}^{e}, \mathcal{D}^{e}, \delta^{e}\right\rangle$, where: $\mathcal{V}^{e}=\mathcal{V} \cup\{e\}, \mathcal{D}^{e}=\mathcal{D}$, and $\delta_{*}^{e}\left(v_{1}^{e}, \ldots, v_{n}^{e}\right)=e$ iff $v_{i}^{e}=e$ for some $v_{i}^{e} \in \mathcal{V}^{e}$. Otherwise, $\delta_{*}^{e}=\delta_{*}$.

Proposition 3.9 Let $M$ be a univalent semantics. Then, for any $\Sigma \cup\{\alpha\}: \Sigma \models_{g}^{M} \alpha$ iff $\Sigma \models_{p}^{M^{e}}$ $\alpha$.

So far we have been looking at the general framework of plurivalent semantics. Here are some examples, obtained by applying plurivalence to the FDE family.

Definition 3.10 Let $M=\langle\mathcal{V}, \mathcal{D}, \delta\rangle$ be a univalent semantics. Then we define a univalent semantics $M^{b}=\left\langle\mathcal{V}^{b}, \mathcal{D}^{b}, \delta^{b}\right\rangle$, where: $\mathcal{V}^{b}=\mathcal{V} \cup\{b\}, \mathcal{D}^{b}=\mathcal{D} \cup\{b\}$, and $\delta_{*}^{b}=\delta_{*}$

Theorem 3.11 Let $M$ be a univalent semantics of the FDE family. Then for any $\Sigma \cup\{\alpha\}$, the following hold: $\Sigma \models_{p}^{M} \alpha$ iff $\Sigma \models_{u}^{M^{b}} \alpha$, and $\Sigma \models_{g}^{M} \alpha$ iff $\Sigma \models_{u}^{M^{e, b}} \alpha$

\subsection{Yet another plurivalent semantics: negative plurivalence}

As is well known, Michael Dunn's discovery in [10] offered an intuitive reading of the truth values in the family of FDE. Seen in this way, Priest's plurivalent semantics offers yet another way of making sense of truth values in terms of smaller number of truth values. But Priest's construction given in 29] did not give any clue to make sense of Weak Kleene Logic and its paraconsistent variant. It turns out, however, that by considering a rather natural variant of Priest's construction, we obtain an intuitive reading of the truth values for those logics.

\footnotetext{
${ }^{10} \mathrm{As}$ an anonymous reviewer points out, since univalently designated values need not be identified with truth, preserving designated values from premises to conclusion, does not collapse with truth-preservation (namely, the preservation of the value $\mathbf{t}$ ).
} 
Definition 3.12 (Negative plurivalent semantics) Given a univalent interpretation, the corresponding negative plurivalent interpretation is the same, except that it replaces the evaluation function, $\mu$, with a one-many evaluation relation, $\mathfrak{R}$, between Prop and $\mathcal{V}$ with the following negativity condition:

$$
\text { for every } p \in \text { Prop: it is not the case that } p \Re v \text { for all } v \in \mathcal{V}
$$

Given an interpretation, $\mathfrak{R}$ is extended to a map from Form to $\mathcal{V}$ recursively:

$$
*\left(\alpha_{1}, \ldots, \alpha_{n}\right) \mathfrak{R} v \text { iff for some } v_{1}, \ldots, v_{n}:\left(\alpha_{i} \mathfrak{R} v_{i} \text { and } v=\delta_{*}\left(v_{1}, \ldots, v_{n}\right)\right)
$$

Finally, we have two definitions of logical consequence in this setting: truth-preservation and non-falsity preservation. In the former case we will say that $\Sigma \models_{n}^{M} \alpha$ iff for all $\mathfrak{R}$, if $\mathfrak{R}$ designates all the formulas of $\Sigma$ then $\mathfrak{R}$ designates $\alpha$, where $\mathfrak{R}$ designates $\alpha$ iff $\alpha \mathfrak{R} v$ for some $v \in \mathcal{D}$. For the latter we will say that $\Sigma \models_{n}^{M} \alpha$ iff for all $\mathfrak{R}$, if $\mathfrak{R}$ designates all the formulas of $\Sigma$ then $\mathfrak{R}$ designates $\alpha$, where $\mathfrak{R}$ designates $\alpha$ iff it is not the case that $\alpha \mathfrak{R} v$ for some $v \notin \mathcal{D}$.

Then, we can again prove a relation between the univalent semantics and negative plurivalent semantics, but the only case that is allowed for the univalent semantics is the two-valued matrix for classical logic. As 22] shows, negative plurivalence does not define a plurivalent consequence relation if some other matrices from the FDE family are taken as the basis. To state the result, the following definition will be useful - alternatively changing the definition of $\dddot{\mathcal{D}}$ to $\dddot{\mathcal{D}}=\{\dddot{v} \in \dddot{\mathcal{V}}: v \notin \ddot{v}$ for all $v \notin \mathcal{D}\}$ for the non-falsity preservation case.

Definition 3.13 Let $M=\langle\mathcal{V}, \mathcal{D}, \delta\rangle$ be the univalent semantics for classical logic. Then we can define a univalent semantics $\dddot{M}=\langle\dddot{\mathcal{V}}, \dddot{\mathcal{D}}, \dot{\delta}\rangle$, where $\dddot{\mathcal{V}}=2^{\mathcal{V}} \backslash \mathcal{V}, \dddot{\mathcal{D}}=\{\dddot{v} \in \dddot{\mathcal{V}}: v \in$ $\ddot{v}$ for some $v \in \mathcal{D}\}$ and

$$
v \in \dddot{\delta}_{*}\left(\dddot{v}_{1}, \ldots, \dddot{v}_{n}\right) \text { iff for some } v_{1}, \ldots, v_{n}:\left(v_{i} \in \dddot{v}_{i} \text { and } v=\delta_{*}\left(v_{1}, \ldots, v_{n}\right)\right) \text {. }
$$

Proposition 3.14 Let $M$ be a univalent semantics for classical logic. Then, for any $\Sigma \cup\{\alpha\}$ : $\Sigma \models \underset{u}{\dddot{M}} \alpha$ iff $\Sigma \models{ }^{\mathbf{w}} \alpha$.

\section{Reflections}

\subsection{On Infectious Values in Plurivalent Semantics}

In the context of both the general and negative plurivalence, following the definitions suggested by Priest, infectious values can only be represented by the empty set. This is remarkably so, even if we start with univalent semantics other than two-valued classical logic, e.g. the threevalued univalent semantics for $\mathbf{K}_{3}^{\mathbf{w}}$, or even FDE. If we, additionally, think of a truth-value $x$ as being true if $\mathbf{t} \in x$, and respectively as being false if $\mathbf{f} \in x$, then it is clear that being true or being false implies being non-infectious ${ }^{11}$

It is for these reasons that if we apply the generalized plurivalence to the e.g. two-valued univalent semantics for classical logic, infectious values cannot be represented with - for instancethe full set $\{\mathbf{t}, \mathbf{f}\}$. We would like to mention, though, that as a remark made by an anonymous reviewer suggests, it will be interesting to discuss definitions of the plurivalent semantics that deviate from Priest's, in order to determine whether or not there is a plurivalent-like setting where, for instance, the full set $\{\mathbf{t}, \mathbf{f}\}$ can represent an infectious value. However, we notice that there is no such thing present in the literature, up to now.

\footnotetext{
${ }^{11}$ Although for an alternative, see [30], where designated infectious values are understood as truth-value gluts, i.e. as both-true-and-false.
} 


\subsection{Addressing the main question}

In what follows we will present an account of the truth conditions for conjunction and disjunction in the context of plurivalent logics which applies both to general plurivalence and negative plurivalence - something that we take to be an advantage of the present discussion.

In the context of infectious logics, interpreted along the lines of $\S 2$, these operations are characterized by the following truth conditions:

$\varphi \wedge \psi$ is true iff $\varphi$ and $\psi$ are true, and condition $C$ applies to $\varphi$ and $\psi$ $\varphi \vee \psi$ is true iff $\varphi$ or $\psi$ are true, and condition $C$ applies to $\varphi$ and $\psi$

where by 'condition $C$ applies to $\varphi$ and $\psi$ ' we mean, respectively, that these sentences are meaningful (in Bochvar's and Halldén's case), that all experts have expressed an opinion toward these sentences (in Fitting's case), that the computer was successful in retrieving the information with regard to these sentences (in Ferguson's case) and that these sentences are on-topic (in Beall's case).

Remark 4.1 Since truth-values in the plurivalent framework are represented as subsets of some set of univalent truth-values, this implies that the curly brackets act as a meaningful operator (if we take into account Bochvar's and Halldén's interpretation), or as a did-expressed-an-opinion operator (if we take into account Fitting's interpretation), or as a successful-in-retrieving value operator (if we take into account Ferguson's interpretation), or as an on-topic operator (if we take into account Beall's interpretation).

On the more conservative side, the traditional account of conjunction has it that a conjunction is true iff both conjuncts are true, whereas the traditional account of disjunction has it that a disjunction is true iff at least one of both disjunctions is true. We will see, through some technical remarks, that this understanding of conjunction is respected in the plurivalent reading of infectious logics, whence we can legitimately say that the operator called 'conjunction' in the context of infectious logic is conjunction. However, the standard understanding of disjunction is not respected in the plurivalent reading of infectious logics, whence we can legitimately say that the operator called 'disjunction' in the context of infectious logics is not disjunction 12

By the truth condition for conjunction in the plurivalent semantics, we have:

$$
\mathbf{t} \in \ddot{\delta}_{\wedge}(x, y) \text { iff } \exists x_{0}, \exists y_{0} \in\{\mathbf{t}, \mathbf{f}\}:\left[x_{0} \in x, y_{0} \in y \text { and } \delta_{\wedge}\left(x_{0}, y_{0}\right)=\mathbf{t}\right] .
$$

But the fact that $\delta_{\wedge}\left(x_{0}, y_{0}\right)=\mathbf{t}$, given the definition of $\delta_{\wedge}$ entails that $x_{0}=\mathbf{t}$ and $y_{0}=\mathbf{t}$, further implying that none of them is the empty set. Thus we have:

$$
\mathbf{t} \in \ddot{\delta}_{\wedge}(x, y) \text { iff } \mathbf{t} \in x \text { and } \mathbf{t} \in y .
$$

From this we infer that conjunction, in the context of infectious logics represented within the plurivalent semantics, is conjunction, as traditionally conceived.

However, we cannot say the same about disjunction, as we now turn to show. By the truth condition for disjunction in the plurivalent semantics, we have:

$$
\mathbf{t} \in \ddot{\delta}_{\vee}(x, y) \text { iff } \exists x_{0}, \exists y_{0} \in\{\mathbf{t}, \mathbf{f}\}:\left[x_{0} \in x, y_{0} \in y \text { and } \delta_{\vee}\left(x_{0}, y_{0}\right)=\mathbf{t}\right] .
$$

Nevertheless, the fact that $\delta_{\vee}\left(x_{0}, y_{0}\right)=\mathbf{t}$, given the definition of $\delta_{\vee}$ does not entail that both $x_{0}, y_{0} \in\{\mathbf{t}, \mathbf{f}\}$, i.e. it does not imply that none of them is the empty set. From which we

\footnotetext{
${ }^{12}$ Notice that we took the notational liberty of using e.g. $\ddot{\delta}_{\wedge}$ as the paradigmatic case, but nothing really depends on this, and $\dddot{\delta} \wedge$ might be used as well, without any loss.
} 
can infer that disjunction, in the context of infectious logics represented within the plurivalent semantics, is not disjunction, as traditionally conceived.

Let us notice, for some readers might be concerned with the case, that negation (as present in infectious logics represented within plurivalent semantics) is negation as traditionally conceived, that is, it is an operator that flip-flops truth and falsity ${ }^{13}$

To conclude, we should highlight that the previous remarks about the degree to which the operators called conjunction and disjunction in infectious logics are legitimately called that way did not make any reference to the validity of inference rules or principles where those connectives are features, e.g. of the already mentioned cases of $\wedge$-Elimination and $\vee$-Introduction.

We would like to point out that those are issues that essentially concern the definition of validity. Whether we do that in terms of truth-preservation, or in terms of non-falsity preservation, in terms of $q$-consequence (cf. [20]) or $p$-consequence (cf. [17]), following its specific instances defined in $\S 2$, the inferences that are going to be valid or invalid vary, as summarized below.

\begin{tabular}{c|cccc} 
& $\models{ }^{\mathbf{K}_{\mathbf{3}}^{\mathbf{w}}}$ & $\models$ PWK & $=_{q}^{\mathbf{W K}}$ & $=_{p}^{\mathbf{W K}}$ \\
\hline$\psi \models \varphi \vee \neg \varphi$ & $\times$ & $\checkmark$ & $\times$ & $\checkmark$ \\
$\varphi \wedge \neg \varphi \models \psi$ & $\checkmark$ & $\times$ & $\times$ & $\checkmark$ \\
$\varphi \models \varphi \vee \psi$ & $\times$ & $\checkmark$ & $\times$ & $\checkmark$ \\
$\varphi \wedge \psi \models \varphi$ & $\checkmark$ & $\times$ & $\times$ & $\checkmark$
\end{tabular}

\section{Conclusion}

In this paper we discussed the extent to which conjunction and disjunction can be rightfully regarded as logical connectives of those particular sorts, in the context of infectious logics. By turning to the analysis of the truth-conditions for these connectives, employing the framework of plurivalent logics, we arrived at the conclusion that - in the context of infectious logicsconjunction is conjunction, whereas disjunction is not disjunction in the context of infectious logics.

There are a number of directions in which further work related to infectious logics and plurivalent logics can be carried out. Regarding the most prominent and historically salient interpretations of infectious logics, in terms of the 'logics of nonsense' due to Bochvar and Halldén, it will be worth exploring the possibility of defining the semantics of a proper meaningful operator within the plurivalent framework. Both Bochvar and Halldén's logics count with such linguistic devices, although in this work we focused mainly on the $\{\neg, \wedge, \vee\}$-fragment of their systems. We leave these and other discussions for a subsequent paper.

\section{References}

[1] Bradley Armour-Garb and Graham Priest. Analetheism: a pyrrhic victory. Analysis, 65(2):167-173, 2005.

[2] Jc Beall. Off-Topic: A New Interpretation of Weak-Kleene Logic. Australasian Journal of Logic, 13(6):136-142, 2016.

[3] Jc Beall and David Ripley. Analetheism and dialetheism. Analysis, 64(1):30-35, 2004.

[4] Nuel Belnap. How a Computer Should Think. In G. Ryle, editor, Contemporary Aspects of Philosophy, pages 30-55. Oriel Press, 1977.

\footnotetext{
${ }^{13}$ For further arguments in favor of the traditional account of negation, see 8 .
} 
[5] Dmitri Bochvar. On a three-valued calculus and its application in the analysis of the paradoxes of the extended functional calculus. Matamaticheskii Sbornik, 4:287-308, 1938.

[6] Ross Brady and Richard Routley. Don't care was made to care. Australasian Journal of Philosophy, 51(3):211-225, 1973.

[7] Charles Daniels. A note on negation. Erkenntnis, 32(3):423-429, 1990.

[8] Michael De and Hitoshi Omori. There is More to Negation than Modality. Journal of Philosophical Logic, 2017.

[9] Harry Deutsch. Paraconsistent analytic implication. Journal of Philosophical Logic, 13(1):1-11, 1984.

[10] Michael Dunn. Intuitive semantics for first-degree entailments and 'coupled trees'. Philosophical Studies, 29(3):149-168, 1976.

[11] Richard Epstein. The Semantic Foundations of Logic, volume I: Propositional Logics. Oxford University Press, New York, 2nd edition, 1995.

[12] Thomas Macaulay Ferguson. A computational interpretation of conceptivism. Journal of Applied Non-Classical Logics, 24(4):333-367, 2014.

[13] Thomas Macaulay Ferguson. Logics of nonsense and Parry systems. Journal of Philosophical Logic, 44(1):65-80, 2015.

[14] Thomas Macaulay Ferguson. Faulty Belnap computers and subsystems of FDE. Journal of Logic and Computation, 26(5):1617-1636, 2016.

[15] Melvin Fitting. Kleene's three valued logics and their children. Fundamenta informaticae, 20(1, 2, 3):113-131, 1994.

[16] Melvin Fitting. Bilattices are nice things. In T. Bolander, V. Hendricks, and S. A. Pedersen, editors, Self-Reference, pages 53-78. CSLI Publications, 2006.

[17] Szymon Frankowski. Formalization of a plausible inference. Bulletin of the Section of Logic, 33(1):41-52, 2004.

[18] Sören Halldén. The Logic of Nonsense. Uppsala Universitets Årsskrift, 1949.

[19] Stephen Cole Kleene. Introduction to metamathematics. North-Holland, Amsterdam, 1952.

[20] Grzegorz Malinowski. Q-consequence operation. Reports on Mathematical Logic, 24(1):49$59,1990$.

[21] John McCarthy. A basis for a mathematical theory of computation. In P. Braffort and D. Hirschberg, editors, Computer Programming and Formal Systems, pages 33-70. NorthHolland Publishing Company, 1963.

[22] Hitoshi Omori. Halldén's Logic of Nonsense and its expansions in view of Logics of Formal Inconsistency. In Proceedings of DEXA2016, pages 129-133. IEEE Computer Society, 2016.

[23] Francesco Paoli. Regressive analytical entailments. Technical Report number 33, Konstanzer Berichte zur Logik und Wissenschaftstheorie, 1992. 
[24] Francesco Paoli. Tautological entailments and their rivals. In J.Y. Béziau, W. Carnielli, and D. Gabbay, editors, Handbook of Paraconsistency, pages 153-175. College Publications, 2007.

[25] William Tuthill Parry. Ein axiomensystem für eine neue art von implikation (analytische implikation). Ergebnisse eines mathematischen Kolloquiums, 4:5-6, 1933.

[26] Graham Priest. Hyper-contradictions. Logique et Analyse, 27(107):237-243, 1984.

[27] Graham Priest. An Introduction to Non-Classical logic: from If to Is. Cambridge University Press, Cambridge, 2nd edition, 2008.

[28] Graham Priest. The logic of the catuskoti. Comparative Philosophy, 1(2):24-54, 2010.

[29] Graham Priest. Plurivalent Logics. The Australasian Journal of Logic, 11(1):1-13, 2014.

[30] Damian Szmuc. An Epistemic Interpretation of Paraconsistent Weak Kleene. Typescript.

[31] Damian Szmuc. Defining LFIs and LFUs in extensions of infectious logics. Journal of Applied Non-Classical Logics, 26(4):286-314, 2016. 